\title{
Periodontal Comparison on Systemic Lupus Erythematosus Patients and Healthy Subjects: A Cross-Sectional Study
}

\author{
'Department of Health, Faculty of Vocational Studies, Universitas Airlangga, Surabaya, Indonesia. \\ ${ }^{2}$ Doctoral Program of Medical Science, Faculty of Medicine, Brawijaya University, Malang, Indonesia. \\ ${ }^{3}$ Department of Clinical Pathology, Faculty of Medicine, Brawijaya University, Malang, Indonesia. \\ ${ }^{4}$ Department of Pharmacology, Faculty of Medicine, Brawijaya University, Malang, Indonesia. \\ ${ }^{5}$ Department of Internal Medicine, Faculty of Medicine, Brawijaya University, Malang, Indonesia.
}

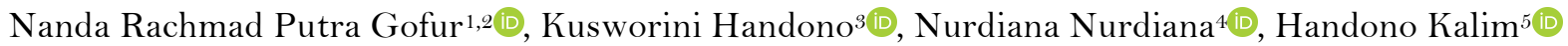

Correspondence: Nanda Rachmad Putra Gofur, Department of Health, Faculty of Vocational Studies, Universitas Airlangga Surabaya, Surabaya Indonesia. E-mail: nanda.rachmad.gofur@,vokasi.unair.ac.id

Academic Editor: Catarina Ribeiro Barros de Alencar

Received: 13 January 2021 / Review: 11 February 2021 / Accepted: 24 February 2021

\begin{abstract}
How to cite: Gofur NBP, Handono K, Nurdiana N, Kalim H. Periodontal comparison on systemic lupus erythematosus patients and healthy subjects: a cross-sectional study. Pesqui Bras Odontopediatria Clín Integr. 2021; $21: e 0006$. https://doi.org/10.1590/pboci.2021.108
\end{abstract}

\begin{abstract}
Objective: To analyze periodontal comparison between Systemic Lupus Erythematosus (SLE) subject and healthy control. Material and Methods: This descriptive cross-sectional study included 122 subjects, 61 SLE patients and 61 healthy subjects who visited the Rheumatology Department, Dr. Saiful Anwar General Hospital, Malang, during 2017-2018. Clinical examination of SLE was using Mexican SLE Disease Activity Index and oral cavity conditions were assessed using the periodontal index, gingival index, calculus index, bleeding on probing, clinical attachment loss and mobility teeth. Results: The age of SLE patients ranged from 18-55 years old with the mean age of $29.50 \pm 9.57$ years old. Periodontitis was higher in SLE patients $(88.5 \%)$ than healthy subjects $(22.95 \%)$. In addition, periodontitis occurrence in SLE $(2.66 \pm 1.02)$ was significantly different $(\mathrm{p}<0.001)$ compared to healthy subjects $(0.51 \pm 0.81)$. Conclusion: This study found higher rates of periodontitis, gingivitis, bleeding on probing, clinical attachment loss, and mobility tooth among SLE patients compared to healthy subjects. Periodontitis was also found to be higher along with more severe SLE group.
\end{abstract}

Keywords: Connective Tissue Diseases; Oral Health; Periodontics; Periodontal Index. 


\section{Introduction}

Periodontal diseases are bacterial infections affecting theperiodontium and beginning from gingival tissue. Furthermore, chronic inflammation resulting in tooth support tissue destruction. Activation of host immune response is usually associated with bacterial, especially gram-negative. Gingivitis is characterized by inflammation of gums without attachment loss. On another side, periodontitis is characterized by the destruction of the hard tissue and soft connective tissue in periodontium. Periodontitis clinical appearance is periodontal pocket formation, loss of connective tissue attachment, alveolar bone resorption, and gingival inflammation [1].

Periodontitis started from gingival inflammation at gingival margin to irreversible damage in the periodontium. Inflammation severity may vary among individuals based on the degree of bacterial infection and dysregulation of host inflammatory response may substantially contribute to its extent. The periodontitis pathway is influenced by systemic conditions from the host, including immune response, anatomical factors, and tissue structural factors [2]. Hence, host conditions could affect periodontitis as a manifestation of certain systemic diseases. Patients with autoimmune disease affect immune system responses resulting in changing accumulation of immune cells, antibodies, metalloproteinase, and cytokines production in blood. Recent studies have shown a potential correlation between periodontitis and autoimmune diseases, especially rheumatoid arthritis and glomerulonephritis [3]. One autoimmune disease that is believed as risk factor for periodontitis is Systemic Lupus Erythematosus (SLE) [4,5].

Oral manifestation is also listed for diagnostic criteria in SLE, specifically the presence of oral ulcers. Previous study found a prevalence of oral manifestations such as xerostomia, dental caries, mucositis, angular cheilitis, ulceration, ranging from $81.3 \%$ to $87.5 \%$, and $93.8 \%$ had periodontitis in SLE patients [6]. On the other hand, it was found that $54.3 \%$ of patients had oral lesions, and $28.1 \%$ presented with ulcers [6]. Worldwide, an increase in the prevalence of periodontitis in SLE has been reported compared to healthy subjects in several studies [7,8]. However, periodontitis comparison between SLE and healthy subjects in Indonesia was never discussed before. Therefore, the aim of this study is to compare periodontitis comparison between SLE and healthy female subjects in Saiful Anwar Hospital, Malang, Indonesia.

\section{Material and Methods}

Ethical Clearance

This study has been approved by ethical committees from Dr. Saiful Anwar General Hospital Malang (No. 400/120/K.3/302/2017). All subjects were required to sign inform consent.

\section{Study Design and Sample}

The design of this study was a descriptive research with a cross-sectional approach. A total of 122 patients were included in this study, 61 SLE and 61 healthy subjects.

All samples were taken from Rheumatology Department Dr. Saiful Anwar General Hospital Malang, Indonesia. Diagnosis of SLE was done by a Rheumatologist in Internal Medicine Consultant based on the 2012 SLICC criteria. Subjects were selected by purposive sampling technique according to the inclusion criteria and exclusion criteria that have been set. Healthy subjects are female subjects without a history of systemic disease. Inclusion SLE criteria were female subjects, age 18-55 with a confirmed SLE diagnosis, willing to become the subject of study, could read and write and had full consciousness. Exclusion criteria were smoking, pregnancy, 
diabetes, and another systemic disease, no past or present history of malignant disease, particularly hematological malignancies, and patients who had hemolytic anemia or defined deficiency of vitamin B12.

In both groups, patient clinical examination of SLE severity using score Mexican SLE Disease Activity and oral cavity were assessed. Evaluated of periodontitis was using periodontal index (PI), gingival index (GI), clinical attachment loss (CAL), bleeding on probing (BOP), plaque index, calculus index and numbers of mobility tooth by Periodontics.

Periodontal Index (PI)

The PI assessment was done by a WHO periodontal examining probe using Periodontal Index by Russel [9]. This index was using the entire surface of the tooth in the oral cavity. Score 0 if there is neither overt inflammation in the investing tissues or loss of function due to destruction of supporting tissue. Score 1 if there is an overt area of inflammation in free gingivae, which does not circumscribe the tooth. Score 2 if inflammation completely circumscribes the tooth, but there is no apparent break in the epithelial attachment. Score 6 if the epithelial attachment has been broken and there is a pocket (not merely a deepened gingival crevice due to swelling in the free gingivae). There is no interference with normal masticatory function; the tooth is firm in its socket and has not drifted. Score 8 if the tooth may be loose; may have drifted; may sound dull on percussion with a metallic instrument; may be depressible in its socket. Total score obtained from total findings divided by all teeth in the oral cavity [10].

\section{Gingival Index (GI)}

The severity of gingival inflammation using gingival index by Loe and Sillness. Measurements on four sides of the gingiva including distal-vestibular papillae, edge of vestibular gingiva, the mesial-vestibular papillae and edge of oral gingiva. Gingival index scores: 0: Normal gingiva; 1: Mild inflammation, slight changes in color and a little edema. There is no bleeding on probing (BOP); 2: Moderate, reddish, edema and shiny inflammation. There is a BOP; 3: Severe inflammation, redness, edema, ulceration and spontaneous bleeding. The gingival index is obtained from the total score of each tooth divided by four sides (4) then divided by total teeth were evaluated [11].

\section{Plaque Index}

Assessments was performed on distal, facial, mesial and lingual surfaces using a mouth glass and a probe. The plaque index was including teeth or replace teeth with tooth restorations or crowns. Only selected teeth can be used in this index. The examination was using six teeth, namely 16, 12, 24, 36, 32 and 44. Plaque Index Assessment of each area was obtained by scoring values of the four surfaces of each tooth and then dividing by four. While the Plaque Index value of each person is obtained by adding up value of each tooth then divided by the number of teeth examined. The following are the Plaque Index assessment criteria [10]: 0 $=$ no plaque in the gingival area; $1=$ thin layer of plaque attached to the edge of the gingiva and its area close to the teeth; 2 = collection of soft deposits that are being accompanied by gingival pockets and at the edge of the gingiva and / or adjacent to the surface of the tooth; $3=$ number of soft deposits accompanied by gingival pockets and / or on gingival edge and adjacent to the tooth surface.

Calculus Index 
Calculus was performed on certain teeth and certain surfaces of these teeth. The maxillary examination consisted of 16 teeth on the buccal surface, 11 on the labial surface and 26 on the labial surface. Whereas the mandibular consists of 36 teeth on the lingual surface, 31 teeth on the labial surface and 46 teeth on the lingual surface: $0=$ On the visible surface of the teeth, there is no calculus or extrinsic staining; $1=$ a. On the visible tooth surface, the calculus covering the tooth surface is $1 / 3$ surface or less than $1 / 3$ surface. b. There are no soft debris on the surface of the teeth, but extrinsic staining that covers the surface of the tooth partially or completely; $2=$ On surface of teeth seen in calculus covering the surface is more than $1 / 3$ of the tooth surface, but less than $2 / 3$ of tooth surface; $3=$ On visible surface of teeth there was calculus covering surface more than $2 / 3$ of surface or the entire surface of tooth.

Bleeding on Probing

Bleeding on probing evaluation by Rebelo and Queiroz [11] is performed on 4 sides of the tooth surface, mesial, distal, buccal and palatal / lingual. If bleeding is found a positive sign, get a score 1. The total amount of bleeding on probing is divided by the number of sides examined, multiplied by $100 \%$. Thus, each individual could have more than 100 sides [11].

\section{Clinical Attachment Loss and Mobility Teeth}

Clinical Attachment Loss (CAL) is the distance between the cementoenamel junction (CEJ) and the periodontal pocket base. The periodontal pocket examination uses a WHO probe; if it has a depth of more than $3.5 \mathrm{~mm}$, the excess was counted as a periodontal pocket. Examination of mobility teeth using two mouth glasses, if there was mobility more than o degree, the total number of teeth was calculated in each patient [11].

Statistical Analysis

The difference two groups were analyzed by Kolmogorov-Smirnoff for normality test, Independent ttest for difference test, and Spearman/Pearson correlation test [12]. The level of significance was set at $\mathrm{p}<0.05$. Statistical analysis was done using SPSS Software, version 21 (IBM SPSS Inc., Chicago, IL, USA).

\section{Results}

A total of 122 subjects were part of this study. The characteristics of the participants are described in Table 1. SLE patients had average ages $29.50 \pm 9.57$ years and healthy subjects $28.57 \pm 9.33$. There was no significant difference in all characteristics among SLE and healthy subjects.

Table 1. Demographic characteristics of participants.

\begin{tabular}{lcc}
\hline \multicolumn{1}{c}{ Variables } & $\begin{array}{c}\text { SLE Patients } \\
\mathrm{N}(\%)\end{array}$ & $\begin{array}{c}\text { Healthy Patients } \\
\mathrm{N}(\%)\end{array}$ \\
\hline Age & $38(62.3)$ & $42(68.8)$ \\
18-30 Years & $23(37.7)$ & $19(32.2)$ \\
$\quad 31-55$ Years & & $50(81.9)$ \\
Ethnics & $49(80.3)$ & $11(18.1)$ \\
$\quad$ Java & $12(19.7)$ & $30(49.2)$ \\
$\quad$ Others & $35(57.3)$ & $31(50.8)$ \\
Marital Status & $26(42.7)$ & \\
$\quad$ Marriage & & $20(32.7)$ \\
$\quad$ Single & $16(26.3)$ &
\end{tabular}




\begin{tabular}{lcc} 
School Graduate & $45(73.7)$ & $41(68.3)$ \\
Employment & & \\
Employed & $20(32.7)$ & $18(29.5)$ \\
Unemployed & $41(68.3)$ & $43(70.5)$ \\
\hline
\end{tabular}

According to the percentage of clinical features between SLE and healthy subjects (Figure 1), we found periodontitis higher in SLE than in healthy individuals (88.50\% versus $22.95 \%)$. Gingivitis was higher in SLE than in healthy individuals (95.08\% versus $65.57 \%)$. Dental plaque was lower in SLE than in healthy individuals (27.86\% versus $68.85 \%$ ). Dental calculus was also lower in SLE than in healthy participants $(9.83 \%$ versus 32.78\%). Clinical Attachment Loss (CAL) was higher in SLE than in healthy individuals (88.5\% versus $22.95 \%)$. The number of teeth with mobility was more elevated in SLE than in healthy subjects (59.01\% versus 9.83\%). Bleeding on Probing was also higher in SLE than in healthy subjects (93.44\% versus $49.18 \%)$.

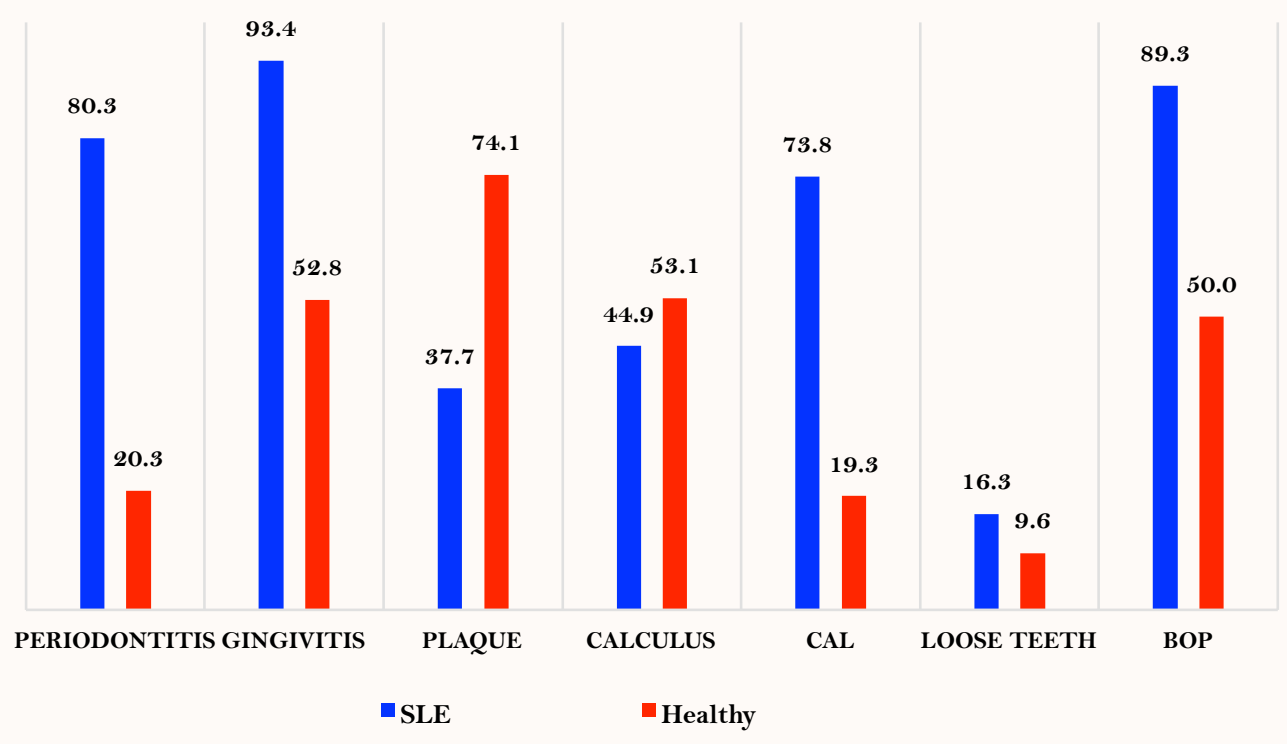

Figure 1. Percentage distribution of clinical characteristics between SLE and healthy subjects.

Periodontitis Comparison between two groups showed in Table 2. There was a significant difference in periodontitis, gingivitis, CAL, mobility teeth and BOP, higher in SLE than healthy subjects $(p<0.001)$. Dental plaque was found significant, but lower in SLE individuals $(p<0.001)$. Dental calculus was found a not significant difference between SLE and healthy subjects $(p=0.065)$.

Table 2. Periodontitis comparison between SLE patients and healthy patients.

\begin{tabular}{lccc}
\hline \multicolumn{1}{c}{ Variables } & SLE Patients & Healthy Patients & p-value \\
\hline Periodontitis & $2.66 \pm 1.02$ & $0.51 \pm 0.81$ & $<0.001$ \\
Gingivitis & $1.95 \pm 1.02$ & $0.83 \pm 0.65$ & $<0.001$ \\
Dental Plaque & $0.53 \pm 0.75$ & $0.90 \pm 0.62$ & $<0.001$ \\
Dental Calculus & $0.35 \pm 0.16$ & $0.64 \pm 0.56$ & 0.065 \\
CAL (mm) & $0.75 \pm 0.59$ & $0.34 \pm 0.55$ & $<0.001$ \\
Mobility Teeth (N) & $1.49 \pm 1.77$ & $0.14 \pm 0.51$ & $<0.001$ \\
BOP (\%) & $42.73 \pm 25.30$ & $11.14 \pm 17.58$ & $<0.001$ \\
\hline
\end{tabular}

Periodontitis comparison in SLE patients, between mild (score 2-5) and severe group (score >5), based SLE Activity Disease Index (SLEDAI), showed in Table 3. There was a significant difference $(\mathrm{p}<0.05)$ between 
the mild and severe groups for periodontitis, gingivitis, dental calculus, CAL, mobility teeth and BOP. However, there was no significant difference in dental plaque between the two groups.

Table 3. Periodontitis comparison in SLE patients with a mild and severe group.

\begin{tabular}{lccc}
\hline \multicolumn{1}{c}{ Variables } & \multicolumn{2}{c}{ Systemic Lupus Erythematosus } & p-value \\
& Mild $(\mathrm{N}=13)$ & Severe $(\mathrm{N}=48)$ & \\
\hline Periodontitis & $1.47 \pm 0.92$ & $2.98 \pm 1.07$ & $<0.001$ \\
Gingivitis & $1.69 \pm 0.85$ & $1.79 \pm 0.94$ & 0.004 \\
Dental Plaque & $0.53 \pm 0.80$ & $0.53 \pm 0.74$ & 0.992 \\
Dental Calculus & $0.87 \pm 0.60$ & $0.67 \pm 0.62$ & 0.009 \\
CAL (mm) & $0.00 \pm 0.00$ & $0.33 \pm 0.72$ & 0.016 \\
Mobility Teeth $(\mathrm{N})$ & $23.92 \pm 19.85$ & $47.83 \pm 24.33$ & 0.002 \\
BOP $(\%)$ & $9.84 \pm 4.14$ & $51.64 \pm 21.80$ & $<0.001$ \\
\hline
\end{tabular}

\section{Discussion}

Periodontitis is a chronic disease involving the destruction of tooth supporting tissue, especially periodontal ligament and alveolar bone. Tissue damage results in bleeding on probing, clinical attachment loss, and mobility teeth. Periodontitis is initiated by a local accumulation of bacteria and sometimes followed by systemic disease. In this case, SLE could worsen periodontal disease. Host response conditions in periodontitis are influenced by bacterial invasion, inflammation responses and immune system [3].

Our results showed significant differences between SLE and health subjects in periodontitis. Previous studied from Ruther-Locher et al. [7], Kobayashi et al. [8] and Rees et al. [14] also reported that SLE has higher periodontitis incidence two times in another country, such as in UK, US, Japan more than healthy subjects. Patients with SLE more likely to have periodontitis due to autoimmune conditions. But, a previous study found no significant difference between SLE patients and healthy subjects. This condition may be due to the small sample size, treatment factor, and another systemic disease. Between the two groups, SLE severity also found a significant difference in periodontitis. Higher disease activity maybe had a significant role in periodontitis higher $[7,13,14]$.

A significant difference was not found between calculus index, suggest that in SLE patients, calculus did not play a significant role in induction periodontitis. Moreover, plaque index was found higher in healthy subject than SLE. Previous study did not prove a correlation with autoimmune diseases, especially SLE and plaque [15]. Association periodontitis in SLE is believed because of dysregulation immune system. Result also a difference in BOP, CAL and mobility tooth. It indicated this periodontitis characteristic with SLE had association and has been proven before $[15,16]$.

Previous studies have suggested response variation is the result of the immune system in inflammation. Polymorphisms of interleukin-1 and interleukin-6 gene were described as the first genetic markers related to periodontitis. Genetic and gene expression studies in patients with SLE have also revealed role of immune system and cytokines that can explain several features of clinical manifestation. Fc receptor has an important role in the regulation of host immune response to bacterial challenge. Polymorphism this receptor related to some autoimmune diseases including SLE [17]. Several studies point to cytokines as important mediators associated with the pathogenesis of periodontitis. Bacterial products induce the synthesis of inflammatory cytokines. Among various cytokines, TNF and IL-1, mediators that can potentially participate in this process. Cytokines stimulate bone resorption by directly inducing the proliferation of osteoclast progenitors and indirectly by stimulating the activity of mature osteoclasts $[18,19]$. 
Theory in SLE subject believed that IL-2, as well as IL-10, TNF $\alpha$, IL-17 and IL-18, levels have been correlated with disease activity and playing a role in Th2 response. Inflammatory cytokines also appear to play an essential role in this relationship. They are often involved in vascular process patients with SLE [20-22]. Changes in local levels of several inflammatory cytokines maybe associated with a possible role in periodontitis. These cytokines are expressed in various cell types, including macrophages, $\mathrm{T}$ lymphocytes, B lymphocytes, and dendritic cells. Studies have shown that cytokine plays a major role in the pathogenesis of various autoimmune diseases, being strongly expressed both local and systemic in adult patients with SLE. Cytokines serum levels were changing in adolescents with SLE compared to healthy subjectd and correlated positively with SLEDAI index [23-25].

Another potential mechanism is the presence of changes in endothelial cells. C-reactive protein contributes to the increasing expression of adhesion molecules cells. Its levels are elevated in patients with periodontitis and autoantibodies directed against this protein and also increasing in SLE patients [26-28]. Elastase, an enzyme in the protease class, also appears to be involved association between periodontal disease and SLE. The infection has been regarded as a triggering factor for autoimmune diseases. It was reported that SLE in children and adults is triggered due to a secondary stimulus such as an infection. The infection could induce abnormal cytokine and immune responses [29,30].

\section{Conclusion}

This study found high rates of periodontitis, gingivitis, bleeding on probing, clinical attachment loss, and missing teeth among SLE patients compared to healthy subjects. But plaque and calculus were found higher in healthy subjects. Periodontitis was also found to be higher along with more severe SLE. It is recommended that further studies need to find the immune pathway between SLE and periodontitis. Furthermore, the results could be used as information on periodontitis in SLE patients.

\section{Authors' Contributions}

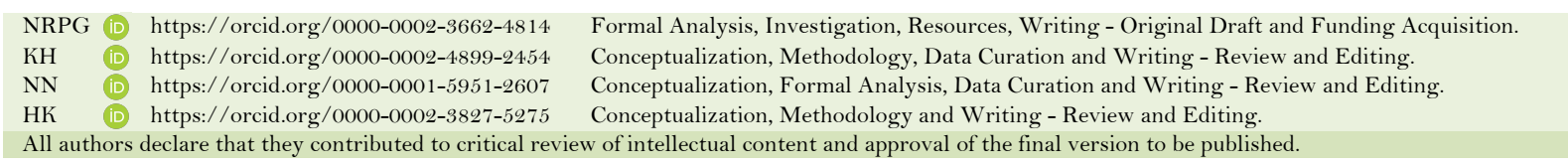

\section{Financial Support}

This research was funded by Indonesian Ministry of Research, Technology and Higher Education (Grant Number 292.47/UN10.C10/PN/2020).

\section{Conflict of Interest}

The authors declare no conflicts of interest.

\section{Data Availability}

The data used to support the findings of this study can be made available upon request to the corresponding author.

\section{References}

[1] Fabbri C, Fuller R, Bonfa E, Guedes LK, D’Alleva PS, Borba EF. Periodontitis treatment improves systemic lupus erythematosus response to immunosuppressive therapy. Clin Rheumatol 2014; 33(4):505-9. https://doi.org/10.1007/s10067-013-2473-2 
[2] Bartold PM, van Dyke TE. Periodontitis: a host-mediated disruption of microbial homeostasis. Unlearning learned concepts. Periodontol 2000 2013; 62(1):203-17. https://doi.org/10.1111/j.1600-0757.2012.00450.x

[3] Cullinan MP, Seymour GJ. Periodontal disease and systemic illness: Will the evidence ever be enough? Periodontol 2000 2013; 62(1):271-86. https://doi.org/10.1111/prd.12007

[4] Genco RJ, Borgnakke WS. Risk factors for periodontal disease. Periodontol 2000 2013; 62(1):59-94. https://doi.org/10.1111/j.1600-0757.2012.00457.x

[5] Ebersole JL, Dawson DR, Morford LA, Peyyala R, Miller, CS, Gonzaléz OA. Periodontal disease immunology: 'Double indemnity' in protecting the host. Periodontol 2000 2013; 62(1):163-202. https://doi.org/10.1111/prd.12005

[6] Calderaro DC, FerreiraGA, Correa JD, Mendonca SM, Silva TA, Costa FO, et al. Is chronic periodontitis premature in systemic lupus erythematosus patients? Clin Rheumatol 2017; 36(3):713-8.

https://doi.org/10.1007/s10067-016-3385-8

[7] Rutter-Locher Z, Fuggle N, Orlandi M, D’Aiuto F, Sofat N. Periodontal Disease and Autoimmunity: What We Have Learned From Microbiome in Rheumatology. In: Arjunan P. Periodontitis - A Useful Reference. London: IntechOpen Limited; 2017.

[8] Kobayashi T, Ito S, Yamamoto K, Hasegawa H, Sugita N, Kuroda T, et al. Risk of periodontitis in systemic lupus erythematosus is associated with Fcy receptor polymorphisms. J Periodontol 2003; 74(3):378-84. https://doi.org/10.1902/jop.2003.74.3.378

[9] Russel AL. The periodontal index. J Periodontol 1967; 38(6P2):585-91. https://doi.org/10.1902/jop.1967.38.6_part2.585

[10] Beltrán-Aguilar ED, Eke PI, Thornton-Evans G, Petersen PE. Recording and surveillance systems for periodontal diseases. Periodontology 2000 2012; 60(1):40-53. https://doi.org/10.1111/j.1600-0757.2012.00446.x

[11] Rebelo MAB, Queiroz AC. Gingival Indices: State of Art. In: Panagakos FS, Davies RM. Gingival Diseases - Their Aetiology, Prevention and Treatment. London: IntechOpen Limited; 2011. pp. 41-54.

[12] Mukaka MM. A guide to appropriate use of correlation coefficient in medical research. Malawi Med J 2012; 24(3):6971.

[13] Albandar JM, Susin C, Hughes FJ. Manifestations of systemic diseases and conditions that affect the periodontal attachment apparatus: Case definitions and diagnostic considerations. J Periodontol 2018; 89(Suppl1):S183-S203. https://doi.org/10.1002/JPER.16-0480

[14] Rees F, Doherty M, Grainge MJ, Lanyon P, Zhang W. The worldwide incidence and prevalence of systemic lupus erythematosus: a systematic review of epidemiological studies. Rheumatology 2017; 56(11):1945-61. https://doi.org/10.1093/rheumatology/kex260

[15] Wu YD, Lin CH, Chao WC, Liao TL, Chen DY, Chen HH. Association between a history of periodontitis and the risk of systemic lupus erythematosus: A nationwide, population-based, case-control study. PloS One 2017; 12(10):e0187075. https://doi.org/10.1371/journal.pone.0187075

[16] Al-Mutairi KD, Al-Zahrani, MS, Bahlas SM, Kahyal RA, Zawawi KH. Periodontal findings in systemic lupus erythematosus patients and healthy controls. Saudi Med J 2015; 36(4):463-8. https://doi.org/10.15537/smj.2015.4.1074

[17] Hammoudeh M, Al-Momani A, Sarakbi H, Chandra P, Hammoudeh S. Oral manifestations of systemic lupus erythematosus patients in Qatar: a pilot study. Int J Rheumatol 2018; 2018:6052326. https://doi.org/10.1155/2018/6052326

[18] Nair S, Faizuddin M, Dharmapalan J. Role of autoimmune responses in periodontal disease. Autoimmune Dis 2014; 2014:596824. https://doi.org/10.1155/2014/596824

[19] Manjarrez-orduño N, Menard LC, Carman JA, Suchard SJ, Casano F, Lee D, et al. A systemic lupus erythematosus endophenotype characterized by increased $\mathrm{CD} 8$ cytotoxic signature associates with renal involvement. ImmunoHorizons 2017; 1(7):124-32.

[20] Kaur G, Mohindra K, Singla S. Autoimmunity: Basics and link with periodontal disease. Autoimmun Rev 2016; 8:714.

[21] Fukushima Y, Minato N, Hattori M. The impact of senescence-associated T cells on immunosenescence and agerelated disorders. Inflamm Regen 2018; 38:24. https://doi.org/10.1186/s41232-018-0082-9

[22] Shah D, Mahajan N, Sah S, Nath SK, Paudyal B. Oxidative stress and its biomarkers in systemic lupus erythematosus. J Biomed Sci 2014; 21(1):18-23.

[23] Perl A, Hanczko R, Doherty E. Assessment of mitochondrial dysfunction in lymphocytes of patients with systemic lupus erythematosus. Methods Mol Biol 2012; 900:61-89. https://doi.org/10.1007/978-1-60761-720-4_4

[24] Gofur NRP, Handono K, Nurdiana N, Kalim H. Association of Th-Tc protein CD28+ and periodontal inflammation among Indonesian women with SLE disease. Sys Rev Pharm 2020; 11 (6):580-6.

[25] Gofur NRP. Impact of SARS-CoV-2 on periodontal tissue manifestation. J Int Oral Health 2020; 12(8):S90-S92.

[26] Handono K, Gofur NRP, Nurdiana, Kalim H, Wahono CS, Poeranto S. Gingivitis on Systemic Lupus Erythematosus (SLE) patients: a pilot study. Res J Pharm Tech 2020; 13(11):5466-70.

https://doi.org/ 10.5958/0974-360X.2020.00954.3 
[27] Gofur NRP, Handono K, Nurdiana N, Kalim H. Periodontitis is associated with disease severity and anti-double stranded DNA antibody and interferon-gamma levels in patients with systemic lupus erythematosus. J Taibah Univ Med Sci 2019; 14(6):560-5. https://doi.org/10.1016/j.jtumed.2019.09.005

[28] Gofur NRP, Nurdiana N, Handono K, Kalim H. Periodontal tissue condition on systemic lupus erythematosus patients: a clinical study. Pesqui Bras Odontopediatria Clín Integr 2020; 20(1):e5094. https://doi.org/10.1590/pboci.2020.010

[29] Gofur NRP, Nurdiana N, Handono K, Kalim H. Immune aging marker associated with periodontitis in systemic lupus erythematosus patients. AIP Conf Proc 2019; 5(1)1-11. https://doi.org/10.1063/1.5109978

[30] Handono K, Gofur NRP, Nurdiana N, Kalim H, Wahono CS, Poeranto S, et al. Role of lymphocytes CD4/CD8 ratio and immunoglobulin $\mathrm{G}$ cytomegalovirus as potential markers for systemic lupus erythematosus patients with periodontal disease. Eur J Dent 2020; 14(4):544-50. https://doi.org/10.1055/s-0040-1715788 